\title{
Stressful life events increase the risk of major depressive episodes: A population-based twin study
}

\author{
Ludvig Daae Bjørndal ${ }^{1,2}$ \& Eivind Ystrom ${ }^{1,2,5}$
}

${ }^{1}$ PROMENTA Research Center, Department of Psychology, University of Oslo, Oslo, Norway

${ }^{2}$ School of Pharmacy, University of Oslo, Oslo, Norway

${ }^{5}$ Department of Mental Disorders, Norwegian Institute of Public Health, Oslo, Norway.

Keywords: stressful life events, depression, twin study, co-twin control, survival analysis 


\begin{abstract}
Previous studies have found that stressful life events (SLEs) are associated with increased risk of adult depression. However, many studies are observational in nature and limited by methodological issues, such as potential confounding by genetic factors. Genetically informative studies, such as the co-twin control design, can strengthen causal inference in observational research. The co-twin control design involves comparing patterns of associations in the full sample and within dizygotic (DZ) and monozygotic twins (MZ). Discrete-time survival analysis has several benefits and multilevel survival analysis can incorporate frailty terms (random effects) to estimate the components of the biometric model. In the current study, we investigated associations between SLEs and depression risk in a population-based twin sample $(\mathrm{N}=2299)$ with a co-twin control design. Associations were modelled using discrete-time survival analysis with biometric frailty terms. SLE occurrence was associated with increased depression risk. Co-twin control analyses indicated that this association was at least in part due to causal influence of SLE exposure on depression risk for event occurrence across all SLEs and of violent SLEs. Stronger within-pair estimates for economic SLEs compared with the full sample association could have resulted if the full sample association was suppressed or if within-pair estimates were inflated. If the former occurred, economic SLEs may represent particularly important risk factors for depression. A minor proportion of the total genetic risk of depression reflected genetic effects related to SLEs. Our findings have implications for future research on SLEs and depression.
\end{abstract}




\section{Introduction}

Stressful life events (SLEs) represent important environmental factors that increase risk of depression (Kessler, 1997; Tennant, 2002). A variety of SLEs have been implicated as risk factors for depression, including assault (Kendler et al., 1999, 2010), job loss (Kendler \& Gardner, 2001) and sexual abuse (Kendler et al., 2000; Kendler \& Aggen, 2014). SLEs that occur during childhood, adolescence and adulthood are all associated with increased risk of depression (Sjöholm et al., 2009; Tennant, 2002).

Research on exposure to risk factors for illness is typically observational in nature due to ethical or practical constraints. Many observational studies are limited by methodological issues, such as potential confounding by genetic or environmental factors. Genetically informative samples can strengthen causal inference in observational research (Pingault et al., 2018). The co-twin control design focuses on twins discordant in exposure to risk factors or outcomes and is one strategy for increasing control over potential confounders.

Findings from several genetically informative studies have indicated that the associations between SLEs and depression in part reflect causal influence of exposure on depression risk (Kendler et al., 1999; Kendler \& Aggen, 2014; Kendler \& Gardner, 2010). However, it has been found that associations may in part reflect non-causal influence or confounding by genetic or familial factors (Brown et al., 2014; Kendler et al., 1999; Silberg et al., 2016). These conflicting findings warrant further studies of the relationship between SLE exposure and depression risk with genetically informative samples.

In the current study, we investigated associations between SLEs and depression in a population-based sample of twins $(\mathrm{N}=2299)$. Three primary research questions were examined: (a) Is SLE occurrence across all SLEs, occurrence of violent SLEs, occurrence of other traumatic SLEs, and/or occurrence of economic SLEs associated with increased depression risk?; (b) can indicated causal patterns of influence of the different grouped SLEs on depression risk be detected in co-twin control analyses?; and (c) how much of the total genetic risk of depression can be explained by genetic effects linked to SLEs?

\section{Methods}

\section{Sample}

Participants were recruited from the Norwegian Institute of Public Health Twin Panel (NIPHTP). The NIPHTP has been described at length elsewhere (Harris et al., 2002, 2006; 
Nilsen et al., 2013). Zygosity was determined by a combination of questionnaire items and genotyping.

Data came from a population-based study of psychiatric disorders among NIPHTP participants with two waves of data collection. From 1999 to 2004 (W1), psychiatric disorders were measured in a subsample of NIPHTP participants. In total, psychiatric interviews were conducted with 2801 twins. Mean age at W1 was 28.2 years (range 19 - 36). At W1, there were $449 \mathrm{MZ}$ female twin pairs, 220 male MZ twin pairs, 263 female DZ twin pairs, 118 male DZ twin pairs, 341 DZ pairs of opposite sex and 19 single twins.

Wave 2 (W2) of data collection was initiated in 2010 (Nilsen et al., 2013). Mean age at W2 was 37.8 years (range $30-44$ ). Clinical diagnostic interviews were completed on 2284 of the twins interviewed during W1. At W2, there were 358 female MZ twin pairs, 154 male MZ twin pairs, 180 female DZ twin pairs, 76 male DZ twin pairs, $219 \mathrm{DZ}$ twin pairs of opposite sex, and 310 single twins. Data on occurrences of SLEs was collected using a questionnaire. In total, 2299 twins responded to the questionnaire at W2.

All participants gave their written informed consent to participate in the study. Approval was sought and received from the Regional Committee for Medical and Health Research Ethics.

\section{Measurements}

Psychiatric interviews were conducted using the using the Munich-Composite International Diagnostic Interview (M-CIDI; Wittchen \& Pfister, 1997). M-CIDI assesses DSM-IV and ICD-10 diagnoses and has good validity and reliability (Reed et al., 1998; Wittchen et al., 1998). Interviewers were primarily clinical psychology students in the final part of their degree or psychiatric nurses (all of whom attended standardised CIDI training). Interviews were largely conducted in person $(8.3 \%$ of the interviews were conducted by telephone for practical reasons) in W1. All interviews were conducted via telephone in W2. Prevalence of DSM-IV Major Depressive Episode (MDE) diagnoses are listed in Table 1.

\section{Table 1}

Depression Diagnoses at Wave 1 and Wave 2

\begin{tabular}{lcc} 
MDE & Wave 1 & Wave 2 \\
\hline Subthreshold & 138 & 91 \\
Threshold & 411 & 406 \\
\hline Total & 549 & 497
\end{tabular}


Subthreshold diagnoses were treated as depressive diagnoses for the primary analyses. Analyses with only threshold diagnoses yielded similar results (see Appendix A).

The SLE questionnaire included 18 SLEs in total. If a participant answered yes for any SLE, he or she was asked to enter the age (in years) when the event was experienced (or when it was first experienced if it had occurred multiple times). The frequencies of all 18 SLEs in the sample are listed in Table 2.

\section{Table 2}

Frequencies of Stressful Life Events

\begin{tabular}{|c|c|c|c|}
\hline Stressful life events (SLEs) & Frequency in full sample & $\begin{array}{c}\text { SLEs before MDE } \\
\text { W1 }\end{array}$ & $\begin{array}{c}\text { SLEs before MDE } \\
\text { W2 }\end{array}$ \\
\hline Life-threatening illness & 125 & 7 & 20 \\
\hline Life-threatening accident & 151 & 17 & 25 \\
\hline $\begin{array}{l}\text { Actively participated in } \\
\text { war/combat }\end{array}$ & 31 & 2 & 6 \\
\hline $\begin{array}{l}\text { Witnessed anyone be badly } \\
\text { injured or killed }\end{array}$ & 229 & 17 & 34 \\
\hline $\begin{array}{l}\text { Threatened with a weapon, } \\
\text { held captive or kidnapped }\end{array}$ & 153 & 15 & 29 \\
\hline $\begin{array}{l}\text { Experienced fire, flooding or } \\
\text { natural disaster }\end{array}$ & 133 & 6 & 23 \\
\hline Rape & 91 & 19 & 26 \\
\hline Sexual abuse & 176 & 51 & 56 \\
\hline $\begin{array}{l}\text { Otherwise physically attacked } \\
\text { or assaulted }\end{array}$ & 303 & 42 & 54 \\
\hline $\begin{array}{l}\text { Otherwise physically abused } \\
\text { as a child }\end{array}$ & 76 & 26 & 28 \\
\hline $\begin{array}{l}\text { Otherwise mistreated as a } \\
\text { child }\end{array}$ & 68 & 19 & 27 \\
\hline $\begin{array}{l}\text { Parental mental illness or } \\
\text { alcohol problems as a child }\end{array}$ & 395 & 105 & 110 \\
\hline $\begin{array}{l}\text { Parental divorce or separation } \\
\text { as a child }\end{array}$ & 451 & 85 & 89 \\
\hline Having divorced or separated & 709 & 29 & 103 \\
\hline $\begin{array}{l}\text { Long-term financial } \\
\text { difficulties }\end{array}$ & 164 & 14 & 27 \\
\hline $\begin{array}{l}\text { Unemployment for more than } \\
\text { six months }\end{array}$ & 236 & 17 & 35 \\
\hline $\begin{array}{l}\text { Major and lasting conflict with } \\
\text { close person }\end{array}$ & 269 & 21 & 40 \\
\hline Other serious life event & 441 & 35 & 70 \\
\hline
\end{tabular}


Note. The table lists the frequency of SLEs in the full sample, SLEs that occurred before a depression diagnosis reported in W1, and SLEs that occurred before a depression diagnosis reported in W2.

The SLEs were aggregated in four groups for statistical analyses. All SLEs included all 18 SLEs listed in Table 2. Violent SLEs included five SLEs: threatened with a weapon, held captive or kidnapped; sexual abuse; rape; otherwise physically attacked or assaulted; and otherwise physically abused as a child. Other traumatic SLEs included three SLEs: lifethreatening accident; actively participated in war/combat; and witnessed anyone be badly injured or killed. Economic SLEs included two SLEs: long-term financial difficulties and unemployment for more than six months.

\section{The biometric model and the co-twin control design}

Twin designs are based on the varying genetic relatedness between monozygotic (MZ) twins, who share $100 \%$ of their genetic material, and dizygotic (DZ) twins, who share $50 \%$ of their genetic material. The basic notion is that, if genetic influences is important for variation in a phenotype, MZ twins will be (on average) more similar than DZ twins for the given phenotype. The biometric model explains phenotypic variation as influenced by additive genetic factors (A), shared environmental factors, non-additive genetic effects (D), shared environmental factors (C) and non-shared environmental factors (E) (Eaves et al., 1978; Fisher, 1918; Jinks \& Fulker, 1970).

The co-twin control design represents one approach to studying exposure to environmental factors that cannot be experimentally manipulated using twin data. The design compares associations in the full sample and for discordant twins. As MZ and DZ twins are matched for early shared environment, and either partially (DZ) or fully (MZ) for genetic material, this increases control over potential confounding by these factors. The co-twin control design can be understood within a counterfactual model of causality (McAdams et al., 2020).

Patterns of associations in the full sample and in within-pair estimates for MZ and DZ twins help discern if environmental exposure may have causal influence on the outcome. If observed associations are equally strong in the full sample and for discordant DZ and MZ twins, this is indicative of causal influence, as within-pair estimates control for shared environmental and genetic factors (partially for DZ twins and fully for MZ twins). Weaker associations within twins compared with the full sample is indicative of confounding by early shared environment. If the associations weaken with genetic similarity (i.e., strongest in the 
full sample and weakest in MZ twins), this is indicative of genetic confounding, which is controlled for to a varying extent in within-pair estimates.

\section{Discrete-time survival analysis}

Associations were modelled using discrete-time survival analysis. Survival analysis has several benefits, including the opportunity to incorporate right-censored observations (i.e., when the time to event is larger than the period of data collection) in estimates of event probabilities and study how risk of events of interest is distributed over time (Hox, 2010). In the discrete-time survival model, a hypothetical coefficient $\beta_{1}$ can be interpreted as the change in the logit of the hazard, following one unit increase in the corresponding covariate. This effect is independent of specific time intervals. Multilevel survival models can incorporate random effects (often called frailty; Austin, 2017).

\section{Statistical analyses}

Analyses were conducted using discrete-time survival analysis with frailty terms to estimate the components of the biometric model. The dataset was converted to a 'long format', which is required for survival analysis (Hox, 2010). SLEs that had occurred after the onset of a depression diagnosis were time-censored. Only first onset MDE diagnoses for each interview were used in the analyses (participants were allowed to report different depressive episodes in W1 and W2).

A parameterisation for between- and within-family effects proposed by Rabe-Hesketh et al. (2008) was applied to estimate the biometric components, with some modifications. Random effects were added at three levels: A random slope at the level of the twin pairs, shared by all twin pairs; a random slope at the level of "zygosity", shared only by MZ twins; and one random effect at the level of participants, capturing residual dependence between measures from the same individual. The variables estimated as random slopes were given the value of $\sqrt{0.5}$. This parameterisation ensures that the co-variance for MZ and DZ twins differs depending on the type of twin.

The described model estimates the AE components. The ACE model was estimated by including an additional random intercept capturing residual dependence between individuals within the same twin pair not captured by genetic effects. The CE model was estimated by including the additional intercept and dropping the Level 2 and Level 3 random 
effects. The ADE model was not estimated as previous studies have not found evidence of non-additive genetic effects for depression (Kendler et al., 1992; Kendler \& Prescott, 1999).

Two constraints were used in the model. The first constrained the residual variance of the random slopes representing genetic effects at Level 2 and Level 3 to be equal. The second constrained the surplus within and the "sparsity" between fixed effects of the DZ twins to be the inverse of each other (these can be constrained to be the inverse of each other as both represent the effects of $0.5 \mathrm{~A})$.

The random effects were added in a generalised linear mixed model (GLMM) with a binomial distribution and a logit link function. The variance of the random effects can be compared against the residual variance (or error) at the lowest-level, which is $\frac{\left(\pi^{2}\right)}{3}$ for the logistic distribution (Hox, 2010). This allows for estimating the biometric components. The intraclass correlation for the models can then be calculated using the estimates of the random effects, i.e., the between estimate divided by the total variance, which is the sum of the between estimate plus the residual variance.

Age of onset was fitted as a fixed effect and inspected up to the third order polynomial, after which it did no longer contribute to model fit. Age of onset, sex and wave of measurement (W1 or W2) were included as fixed effects. The dependent variable was having received a depression diagnosis at any time point. Models were evaluated using AIC (Akaike, 1987). The ACE model did not converge to estimate a confidence interval for the intercept estimating the $\mathrm{C}$ component $(A I C=8715.20)$. The $\mathrm{C}$ component could be dropped without deterioration in model fit for the $\mathrm{AE}$ model $(A I C=8715.20)$. The $\mathrm{CE}$ model did not improve model fit $(A I C=8719.59)$. Therefore, the AE model served as the basis for subsequent models that incorporated the effects of SLEs. 


\section{Results}

\section{Model with no SLEs}

Table 3 reports the results for the fixed and random effects in the AE model with no SLEs.

\section{Table 3}

Fixed and Random Effects of the AE Model

\begin{tabular}{lcccl} 
Fixed part & $\beta$ & $S E$ & $\mathrm{p}$ & $95 \%$ Confidence Interval \\
\hline Age of onset $(t)$ & .4619 & .0844 & .0000 & {$[.2965, .6273]$} \\
Age of onset $^{2}$ & -.0112 & .0037 & .0030 & {$[-.0184,-.0039]$} \\
Age of onset $^{3}$ & .0001 & .0001 & .0580 & {$[-.0000, .0002]$} \\
Wave 2 & -.5721 & .0860 & .0000 & {$[-.7406,-.4036]$} \\
Sex (female) & .6317 & .1216 & .0000 & {$[.3934, .8700]$} \\
Intercept & -11.1380 & .6365 & .0000 & {$[-12.3855,-9.8905]$} \\
\hline Random part & & & \\
\hline$\sigma_{\mathrm{A}}$ & 1.3297 & .2453 & & {$[.9263,1.9089]$} \\
$\sigma_{\mathrm{E}}$ & .9457 & .2600 & & {$[.5518,1.6208]$}
\end{tabular}

The fixed effects of time $t$ determine the baseline hazard rate, i.e., the hazard when all covariates are zero (Rabe-Hesketh \& Skrondal, 2012). Risk of depression increased over time, but was slightly reduced at later time points (i.e., older ages) in the sample. The hazard rate peaked around age 30 and depression risk was higher for females.

The heritability estimate for receiving a first subthreshold or threshold MDE diagnosis in any given year was $24 \%$. By excluding the lowest-level error term, the heritability of lifetime risk of depression was estimated at 58\%. This latter approach is asymptotic to the "measurement model" of psychometrics, where random measurement error is excluded from effect estimates (Kendler et al., 1993; van den Berg et al., 2007).

Stable and unstable environmental effects on depression risk were disentangled by dividing the estimated $\sigma_{E}$ and the lowest-level variance by the total variance. In any given year in the current sample, depression risk was explained by $24 \%$ genetic influences, $17 \%$ stable (time-invariant) environmental influences, and 59\% unstable (time-variant) environmental influences including measurement error.

\section{Co-twin control analyses}


Table 4 reports the results of co-twin control analyses of associations between grouped SLEs and depression risk.

\section{Table 4}

Results of Co-twin Control Analyses Displaying hORs

\begin{tabular}{lcccccc} 
& \multicolumn{2}{c}{ Full sample } & \multicolumn{2}{c}{ Within DZ twins } & \multicolumn{2}{c}{ Within MZ twins } \\
\cline { 2 - 7 } $\begin{array}{l}\text { Stressful life } \\
\text { events }\end{array}$ & hOR $(S E)$ & $\mathrm{p}$ & $\mathrm{hOR}(S E)$ & $\mathrm{p}$ & $\mathrm{hOR}(S E)$ & $\mathrm{p}$ \\
\hline All SLEs & $1.383(.046)$ & .000 & $1.398(.151)$ & .002 & $1.423(.114)$ & .000 \\
Violent SLEs & $1.551(.114)$ & .000 & $1.310(.333)$ & .287 & $1.430(.238)$ & .032 \\
$\begin{array}{l}\text { Other traumatic } \\
\text { SLEs }\end{array}$ & $1.176(.172)$ & .267 & $.711(.336)$ & .470 & $.784(.245)$ & .436 \\
Economic SLEs & $2.021(.291)$ & .000 & $3.307(1.823)$ & .030 & $3.103(1.043)$ & .001
\end{tabular}

Note. hOR refers to hazard odds ratio (Petras et al., 2011). Associations were statistically significant if corresponding $\mathrm{p}$-values were below a threshold of $\alpha=.05$

Occurrence of one SLE (across all 18 SLEs) was associated with increased depression risk in the full sample $(95 \% \mathrm{CI}[1.296,1.475])$. The associations were similar in size for DZ twins (95\% CI [1.131, 1.727]) and MZ twins (95\% CI [1.216, 1.664]).

For violent SLEs, occurrence of one SLE was associated with increased depression risk in the full sample (95\% CI $[1.343,1.790])$ and for MZ twins $(95 \%$ CI $[1.032,1.982])$. The within-pair association for DZ twins was slightly attenuated compared with the withinpair estimate MZ twins and was not statistically significant (95\% CI [.797, 2.155]).

For other traumatic SLEs, occurrence of one SLE was not associated with increased risk of depression in the full sample (95\% CI [.883, 1.567]), nor for MZ twins (95\% CI [.425, $1.445])$ or DZ twins (95\% CI [.282, 1.793]).

For economic SLEs, occurrence of one event was associated with increased depression risk in the full sample (95\% CI [1.524, 2.681]), and stronger associations were observed for MZ twins (95\% CI [1.606, 5.995]) and for DZ twins (95\% CI [1.122, 9.744]).

Table 5 displays the residual random effects estimated in the models incorporating the various SLEs. Heritability was estimated before and after controlling for SLEs. The reduction in $\sigma_{\mathrm{A}}$ after including SLEs reflects how much of the total genetic risk of depression which was explained by genetic effects linked to SLEs, in the present sample. After including all of the SLEs, heritability of depression went from $58 \%$ to $50 \%$. 


\section{Table 5}

Residual Random Effects for the Models With Grouped SLEs

\begin{tabular}{lcccc} 
Grouped SLEs & $\sigma_{A}$ & $\sigma_{E}$ & $\mathrm{~h}^{2}$ & Reduction in $\mathrm{h}^{2}$ after including SLEs \\
\hline Baseline model & 1.383 & 0.946 & 0.584 & \\
All SLEs & 1.031 & 1.039 & 0.498 & $14.8 \%$ \\
Violent SLEs & 1.152 & 0.928 & 0.554 & $5.2 \%$ \\
Other traumatic SLEs & 1.307 & 0.966 & 0.575 & $1.6 \%$ \\
Economic SLEs & 1.239 & 0.949 & 0.566 & $3.1 \%$
\end{tabular}

Note. Table 5 displays residual random effects in the AE models incorporating the effects of SLEs and the reduction in additive genetic effects after including SLEs in the models (in percentages). These were compared to the random effects estimated in the AE model with no SLEs, listed in Table 3.

\section{Discussion}

Overall, SLE occurrence was associated with increased depression risk for all 18 SLEs, violent SLEs and economic SLEs in the full sample. For all SLEs and violent SLEs, co-twin control analyses indicated that these associations at least in part reflect causal influence of exposure on depression risk. This finding is in accordance with previous genetically informative studies that have found that the association between dependent SLEs and depression risk is substantially causal in nature (Kendler et al., 1999; Kendler \& Gardner, 2010). Almost all events in the current study were personal SLEs (i.e., SLEs that occur to individuals): indicated causal influence of exposure to personal SLEs on depression risk has also been found previously (Kendler et al., 1999). Occurrence of various forms of trauma has also been found to have a causal influence on depression risk (Brown et al., 2014; McCutcheon et al., 2009).

Indicated causal patterns of influence on depression risk has been found for a number of specific SLEs included in the current study as well, such as combat exposure (Koenen et al., 2003), assault (Kendler et al., 1999, 2010), sexual abuse (Kendler et al., 2000; Kendler \& Aggen, 2014; Nelson et al., 2002), and romantic problems and job loss (Kendler \& Gardner, 2001). Evidence of a causal effect of maltreatment in childhood on risk of major depression was also found in a recent GWAS meta-analysis using Mendelian Randomisation (Warrier et al., 2021).

No associations between the occurrence of other traumatic SLEs and depression risk were observed in the current study. This diverges from previous genetically informative studies, which have found increased depression risk associated with combat exposure 
(Koenen et al., 2003) and somatic illness and injury (Kendler et al., 1999). While it is not clear why there is an absence of associations between other traumatic SLEs and depression risk in the current study, we note that this SLE grouping included fewer SLEs compared with violent and all SLEs and was substantially more heterogeneous compared with the other aggregated SLEs.

For economic SLEs, strong associations were observed between event occurrence and increased risk of depression in the full sample. This is in line with previous genetically informative studies that have found increased depression risk associated with financial problems and job loss (Kendler et al., 1999, 2010; Kendler \& Gardner, 2001, 2014).

However, observed associations between occurrence of economic SLEs and depression risk were stronger in within-pair estimates for $\mathrm{MZ}$ and $\mathrm{DZ}$ twins compared with the full sample. Two potential explanations for this pattern of associations are proposed. Firstly, it may be the case that this association was suppressed in the full sample due to confounding. This could have resulted if there was an inverse effect of the confounder(s) on exposure and outcome, which masked or suppressed an effect in the full sample (e.g., if familial factors associated with less economic problems were associated with increased depression risk, this could suppress the full sample association). This possibility implies that the association could be underestimated in the full sample and that economic SLEs may be a particularly important risk factor for depression in the current sample.

Several previous genetically informative studies have implicated economic SLEs as risk factors for depression, but not specifically reported on within-pair estimates of the associations (Kendler et al., 1999, 2010; Kendler \& Halberstadt, 2013). However, findings from some previous genetically informative studies indicate that this association is at least in part causal. Using a similar design as in the current study, Kendler \& Gardner (2001) found that $\mathrm{MZ}$ twins with a history of depression reported higher rates of job loss compared with non-depressed co-twins. Kendler \& Gardner (2014) found that the association between financial and occupational difficulties and depression risk were stronger in male twins discordant for depression compared with female twins.

A second possible explanation is that the within-pair associations were inflated due to non-shared confounders in twin pairs. Stronger associations in within-pair estimates than the full sample can result when confounding variable(s) within twin pairs have lower correlation than the correlation of exposure to a risk factor within twin pairs (Frisell et al., 2012). As discordant twins are selectively chosen to be dissimilar, this implies that they differ in nonshared causes of exposure to risk factors (i.e., a twin pair discordant for exposure is likely 
less similar than a twin pair concordant for exposure). If a confounder increases the probability of exposure to economic SLEs and depression, an unexposed twin would be less likely to possess the confounder and become depressed, as the confounder increases the risk of depression. This can potentially inflate within-pair estimates.

\section{Limitations}

The current study suffers from several limitations. Firstly, although the co-twin control design increases control over genetic and shared environmental confounders, it is unable to control for non-shared confounders that only affect one twin, which can bias estimated effects (Frisell et al., 2012; Kendler \& Gardner, 2010). Secondly, we focused on main effects of SLEs, however previous studies have found genotype-environment interactions for SLEs and depression (e.g., Choi et al., 2020; Mandelli \& Serretti, 2013). Such interactions may be present in the current sample, but were beyond the scope of the current study to investigate. Thirdly, the current sample was large and population-based, but consisted only of young adults. Thus, risk of depression could not be investigated in later adulthood. In addition, substantial participant loss from W1 to W2 may have induced some attrition bias (i.e., systematic loss of participants). Fourthly, the current study was retrospective in nature. Therefore, the possible influence of recall bias (i.e., that current depression or mood states affects correct reporting of previously experienced SLEs or depressive episodes) cannot be excluded. Fifth, participants were censored after the first onset of a MDE, and so we did not study the effects of SLEs on recurrent depression.

\section{Implications}

The findings of the current study have several implications. The results support previous research in implicating SLEs as important risk factors with probable causal influence on depression risk. If the full sample association for economic SLEs was suppressed, the effects of economic SLEs on depression may be stronger than what has previously been found. Future studies should investigate if this association was suppressed or if within-pair estimates were inflated, also by using longitudinal designs.

Efforts to prevent depression, which have been called for (Cuijpers et al., 2012), should also consider aiming to prevent SLEs. The SLEs of the current study encompass a variety of stressors, many of which can be intervened on and prevented.

When controlling for SLE occurrence (across all 18 SLEs), the estimated heritability of depression was slightly reduced, indicating that a minor proportion of the genetic risk of 
depression reflected genetic effects linked to SLEs. This estimate aligns with that of one previous study, which found that roughly $10-15 \%$ of the impact of genetic risk of depression was mediated through life experiences (Kendler \& Karkowski-Shuman, 1997). Minor genetic effects linked to SLEs suggest that it is unlikely that a large proportion of identified genetic variants will be linked to SLEs in GWASes. This also illustrates the utility of quantitative genetic designs in estimating genetic factors with direct effects and genetic factors acting through a heritable exposure.

\section{Conclusion}

In the current study, we investigated patterns of associations between exposure to SLEs and depression risk in a large population-based twin sample using a co-twin control design. Depression diagnoses were based on repeated clinical interviews across two waves of measurements. Event occurrence across all 18 SLEs, of violent SLEs and of economic SLEs was associated with increased risk of depression. For the former two, co-twin control analyses indicated that the associations at least in part reflect causal influence of SLE exposure on depression risk. The pattern of associations for economic SLEs could have resulted from suppression of the full sample association or inflated within-estimates due to non-shared confounding. If the association was suppressed in the full sample, economic SLEs may be particularly important risk factors for depression. No associations between other traumatic SLEs and depression risk were observed. A minor proportion of the total genetic risk of depression reflected genetic effects related to SLEs.

\section{References}

Akaike, H. (1987). Factor analysis and AIC. Psychometrika, 52(3), 317-332. https://doi.org/10.1007/BF02294359

Austin, P. C. (2017). A tutorial on multilevel survival analysis: Methods, models and applications. International Statistical Review, 85(2), 185-203. https://doi.org/10.1111/insr.12214

Brown, R. C., Berenz, E. C., Aggen, S. H., Gardner, C. O., Knudsen, G. P., ReichbornKjennerud, T., Kendler, K. S., \& Amstadter, A. B. (2014). Trauma exposure and Axis I psychopathology: A co-twin control analysis in Norwegian young adults. 
Psychological Trauma: Theory, Research, Practice, and Policy, 6(6), 652-660. https://doi.org/10.1037/a0034326

Choi, K. W., Chen, C.-Y., Ursano, R. J., Sun, X., Jain, S., Kessler, R. C., Koenen, K. C., Wang, M.-J., Wynn, G. H., Major Depressive Disorder Working Group of the Psychiatric Genomics Consortium, Campbell-Sills, L., Stein, M. B., \& Smoller, J. W. (2020). Prospective study of polygenic risk, protective factors, and incident depression following combat deployment in US Army soldiers. Psychological Medicine, 50(5), 737-745. https://doi.org/10.1017/S0033291719000527

Cuijpers, P., Beekman, A. T. F., \& Reynolds, C. F. (2012). Preventing depression: A global priority. JAMA, 307(10), 1033-1034. https://doi.org/10.1001/jama.2012.271

Eaves, L. J., Last, K. A., Young, P. A., \& Martin, N. G. (1978). Model-fitting approaches to the analysis of human behaviour. Heredity, 41(3), 249-320. https://doi.org/10.1038/hdy.1978.101

Fisher, R. A. (1918). The correlation between relatives on the supposition of Mendelian inheritance. Earth and Environmental Science Transactions of The Royal Society of Edinburgh, 52(2), 399-433. https://doi.org/10.1017/S0080456800012163

Frisell, T., Öberg, S., Kuja-Halkola, R., \& Sjölander, A. (2012). Sibling comparison designs: Bias from non-shared confounders and measurement error. Epidemiology, 23(5), $713-$ 720. https://doi.org/10.1097/EDE.0b013e31825fa230

Harris, J. R., Magnus, P., \& Tambs, K. (2002). The Norwegian Institute of Public Health Twin Panel: A description of the sample and program of research. Twin Research and Human Genetics, 5(5), 415-423. https://doi.org/10.1375/twin.5.5.415

Harris, J. R., Magnus, P., \& Tambs, K. (2006). The Norwegian Institute of Public Health Twin Program of Research: An update. Twin Research and Human Genetics, 9(6), 858-864. https://doi.org/10.1375/twin.9.6.858 
Hox, J. J. (2010). Multilevel analysis: Techniques and applications (2nd ed.). Routledge.

Jinks, J. L., \& Fulker, D. W. (1970). Comparison of the biometrical genetical, MAVA, and classical approaches to the analysis of the human behavior. Psychological Bulletin, 73(5), 311-349. https://doi.org/10.1037/h0029135

Kendler, K. S., \& Aggen, S. H. (2014). Clarifying the causal relationship in women between childhood sexual abuse and lifetime major depression. Psychological Medicine, 44(6), 1213-1221. https://doi.org/10.1017/S0033291713001797

Kendler, K. S., Bulik, C. M., Silberg, J., Hettema, J. M., Myers, J., \& Prescott, C. A. (2000). Childhood sexual abuse and adult psychiatric and substance use disorders in women: An epidemiological and cotwin control analysis. Archives of General Psychiatry, 57(10), 953-959. https://doi.org/10.1001/archpsyc.57.10.953

Kendler, K. S., \& Gardner, C. O. (2001). Monozygotic twins discordant for major depression: A preliminary exploration of the role of environmental experiences in the aetiology and course of illness. Psychological Medicine, 31(3), 411-423. https://doi.org/10.1017/S0033291701003622

Kendler, K. S., \& Gardner, C. O. (2010). Dependent stressful life events and prior depressive episodes in the prediction of major depression: The problem of causal inference in psychiatric epidemiology. Archives of General Psychiatry, 67(11), 1120-1127. https://doi.org/10.1001/archgenpsychiatry.2010.136

Kendler, K. S., \& Gardner, C. O. (2014). Sex differences in the pathways to major depression: A study of opposite-sex twin pairs. American Journal of Psychiatry, 171(4), 426-435. https://doi.org/10.1176/appi.ajp.2013.13101375

Kendler, K. S., \& Halberstadt, L. J. (2013). The road not taken: Life experiences in monozygotic twin pairs discordant for major depression. Molecular Psychiatry, 18(9), 975-984. https://doi.org/10.1038/mp.2012.55 
Kendler, K. S., Karkowski, L. M., \& Prescott, C. A. (1999). Causal relationship between stressful life events and the onset of major depression. American Journal of Psychiatry, 156(6), 837-841. https://doi.org/10.1176/ajp.156.6.837

Kendler, K. S., \& Karkowski-Shuman, L. (1997). Stressful life events and genetic liability to major depression: Genetic control of exposure to the environment? Psychological Medicine, 27(3), 539-547. https://doi.org/10.1017/S0033291797004716

Kendler, K. S., Kessler, R. C., Walters, E. E., MacLean, C. J., Neale, M. C., Heath, A. C., \& Eaves, L. J. (2010). Stressful life events, genetic liability, and onset of an episode of major depression in women. FOCUS, 8(3), 459-470. https://doi.org/10.1176/foc.8.3.foc459

Kendler, K. S., Neale, M. C., Kessler, R. C., Heath, A. C., \& Eaves, L. J. (1992). A population-based twin study of major depression in women: The impact of varying definitions of illness. Archives of General Psychiatry, 49(4), 257-266. https://doi.org/10.1001/archpsyc.1992.01820040009001

Kendler, K. S., Neale, M. C., Kessler, R. C., Heath, A. C., \& Eaves, L. J. (1993). The lifetime history of major depression in women: Reliability of diagnosis and heritability. Archives of General Psychiatry, 50(11), 863-870. https://doi.org/10.1001/archpsyc.1993.01820230054003

Kendler, K. S., \& Prescott, C. A. (1999). A population-based twin study of lifetime major depression in men and women. Archives of General Psychiatry, 56(1), 39-44. https://doi.org/10.1001/archpsyc.56.1.39

Kessler, R. C. (1997). The effects of stressful life events on depression. Annual Review of Psychology, 48, 191-214. https://doi.org/10.1146/annurev.psych.48.1.191

Koenen, K. C., Lyons, M. J., Goldberg, J., Simpson, J., Williams, W. M., Toomey, R., Eisen, S. A., True, W., \& Tsuang, M. T. (2003). Co-twin control study of relationships 
among combat exposure, combat-related PTSD, and other mental disorders. Journal of Traumatic Stress, 16(5), 433-438. https://doi.org/10.1023/A:1025786925483

Mandelli, L., \& Serretti, A. (2013). Gene environment interaction studies in depression and suicidal behavior: An update. Neuroscience and Biobehavioral Reviews, 37(10), 2375-2397. https://doi.org/10.1016/j.neubiorev.2013.07.011

McAdams, T. A., Rijsdijk, F. V., Zavos, H. M. S., \& Pingault, J.-B. (2020). Twins and causal inference: Leveraging nature's experiment. Cold Spring Harbor Perspectives in Medicine. https://doi.org/10.1101/cshperspect.a039552

McCutcheon, V. V., Heath, A. C., Nelson, E. C., Bucholz, K. K., Madden, P. A. F., \& Martin, N. G. (2009). Accumulation of trauma over time and risk for depression in a twin sample. Psychological Medicine, 39(3), 431-441. https://doi.org/10.1017/S0033291708003759

Nelson, E. C., Heath, A. C., Madden, P. A. F., Cooper, M. L., Dinwiddie, S. H., Bucholz, K. K., Glowinski, A., McLaughlin, T., Dunne, M. P., Statham, D. J., \& Martin, N. G. (2002). Association between self-reported childhood sexual abuse and adverse psychosocial outcomes: Results from a twin study. Archives of General Psychiatry, 59(2), 139-145. https://doi.org/10.1001/archpsyc.59.2.139

Nilsen, T. S., Knudsen, G. P., Gervin, K., Brandt, I., Røysamb, E., Tambs, K., Ørstavik, R., Lyle, R., Reichborn-Kjennerud, T., Magnus, P., \& Harris, J. R. (2013). The Norwegian Twin Registry from a public health perspective: A research update. Twin Research and Human Genetics, 16(1), 285-295. https://doi.org/10.1017/thg.2012.117

Petras, H., Masyn, K. E., Buckley, J. A., Ialongo, N. S., \& Kellam, S. (2011). Who is most at risk for school removal? A multilevel discrete-time survival analysis of individualand context-level influences. Journal of Educational Psychology, 103(1), 223-237. https://doi.org/10.1037/a0021545 
Peyrot, W. J., Milaneschi, Y., Abdellaoui, A., Sullivan, P. F., Hottenga, J. J., Boomsma, D. I., \& Penninx, B. W. J. H. (2014). Effect of polygenic risk scores on depression in childhood trauma. The British Journal of Psychiatry, 205(2), 113-119. https://doi.org/10.1192/bjp.bp.113.143081

Pingault, J.-B., O’Reilly, P. F., Schoeler, T., Ploubidis, G. B., Rijsdijk, F., \& Dudbridge, F. (2018). Using genetic data to strengthen causal inference in observational research. Nature Reviews Genetics, 19(9), 566-580. https://doi.org/10.1038/s41576-018-0020-3

Rabe-Hesketh, S., \& Skrondal, A. (2012). Multilevel and longitudinal modeling using Stata (3rd ed., Vol. 2). Stata Press.

Rabe-Hesketh, S., Skrondal, A., \& Gjessing, H. K. (2008). Biometrical modeling of twin and family data using standard mixed model software. Biometrics, 64(1), 280-288. https://doi.org/10.1111/j.1541-0420.2007.00803.x

Reed, V., Gander, F., Pfister, H., Steiger, A., Sonntag, H., Trenkwalder, C., Sonntag, A., Hundt, W., \& Wittchen, H.-U. (1998). To what degree does the Composite International Diagnostic Interview (CIDI) correctly identify DSM-IV disorders? Testing validity issues in a clinical sample. International Journal of Methods in Psychiatric Research, 7(3), 142-155. https://doi.org/10.1002/mpr.44

Silberg, J. L., Copeland, W., Linker, J., Moore, A. A., Roberson-Nay, R., \& York, T. P. (2016). Psychiatric outcomes of bullying victimization: A study of discordant monozygotic twins. Psychological Medicine, 46(9), 1875-1883. https://doi.org/10.1017/S0033291716000362

Sjöholm, L., Lavebratt, C., \& Forsell, Y. (2009). A multifactorial developmental model for the etiology of major depression in a population-based sample. Journal of Affective Disorders, 113(1-2), 66-76. https://doi.org/10.1016/j.jad.2008.04.028 
Tennant, C. (2002). Life events, stress and depression: A review of recent findings. Australian \& New Zealand Journal of Psychiatry, 36(2), 173-182. https://doi.org/10.1046/j.1440-1614.2002.01007.x

van den Berg, S. M., Glas, C. A. W., \& Boomsma, D. I. (2007). Variance decomposition using an IRT measurement model. Behavior Genetics, 37(4), 604-616. https://doi.org/10.1007/s10519-007-9156-1

Warrier, V., Kwong, A., Luo, M., Dalvie, S., Croft, J., Sallis, H., Baldwin, J., Munafo, M., Nievergelt, C., Grant, A., Burgess, S., Moore, T., Barzilay, R., McIntosh, A., Marinus, van Ij., \& Cecil, C. (2021). Gene-environment correlations and causal effects of childhood maltreatment on physical and mental health: A genetically informed approach. The Lancet, 1-14. https://doi.org/10.17863/CAM.62469

Wittchen, H.-U., Lachner, G., Wunderlich, U., \& Pfister, H. (1998). Test-retest reliability of the computerized DSM-IV version of the Munich-Composite International Diagnostic Interview (M-CIDI). Social Psychiatry and Psychiatric Epidemiology, 33(11), 568578.

Wittchen, H.-U., \& Pfister, H. (1997). DIA-X Interview (M-CIDI). Swets \& Zeitlinger. 


\section{Appendix A}

Appendix A reports the output for models run with only threshold MDE diagnoses. These yield very similar estimated associations as the models reported in the article (which used both subthreshold and threshold diagnoses).

\section{Table A1}

AE Model With All 18 SLEs and Only Threshold MDE Diagnoses

\begin{tabular}{lcccl} 
Fixed part & $\beta$ & $S E$ & $\mathrm{p}$ & 95\% Confidence Interval \\
\hline SLE_tot_cm & .3679 & .0459 & .0000 & {$[.2780, .4578]$} \\
SLE_tot_cm\#c.dz & .0107 & .0522 & .8380 & {$[-.0916, .1129]$} \\
SLE_tot_cmc & .3302 & .0768 & .0000 & {$[.1798, .4807]$} \\
SLE_tot_cmc\#c.dz & -.0107 & .0522 & .8380 & {$[-.1129, .0916]$} \\
$\mathrm{t}$ & .4440 & .0867 & .0000 & {$[.2741, .6141]$} \\
$\mathrm{t}^{2}$ & -.0116 & .0037 & .0010 & {$[-.0188,-.0044]$} \\
$\mathrm{t}^{3}$ & .0001 & .0000 & .0290 & {$[.0000, .0002]$} \\
$\mathrm{W}$ & -.4223 & .0853 & .0000 & {$[-.5896,-.2551]$} \\
Sex & .6338 & .1219 & .0000 & {$[.3949, .8727]$} \\
_cons & -11.6438 & .6760 & .0000 & {$[-12.9688,-10.3188]$} \\
\hline Random part & & & & \\
\hline$\sigma_{\mathrm{A}}$ & 1.2349 & .2677 & & {$[.8074,1.888]$} \\
$\sigma_{\mathrm{E}}$ & 1.220 & .299 & & {$[.7543,1.9729]$}
\end{tabular}

$\mathrm{AIC}=8788.4510(\mathrm{df}=11)$ 


\section{Table A2}

AE Model With Violent SLEs and Only Threshold MDE Diagnoses

\begin{tabular}{lcccl} 
Fixed part & $\beta$ & $S E$ & $\mathrm{p}$ & 95\% Confidence Interval \\
\hline violent_cm & .5695 & .1093 & .0000 & {$[.3552 .7837]$} \\
violent_cm\#c.dz & -.0140 & .1301 & .9140 & {$[-.2690, .2409]$} \\
violent_cmc & .3277 & .1554 & .0350 & {$[.0232, .6323]$} \\
violent_cmc\#c.dz & .0140 & .1301 & .9140 & {$[-.2409, .2690]$} \\
$\mathrm{t}$ & .4445 & .0861 & .0000 & {$[.2758, .6132]$} \\
$\mathrm{t}^{2}$ & -.0113 & .0036 & .0020 & {$[-.0184,-.0042]$} \\
$\mathrm{t}^{3}$ & .0001 & .0000 & .0310 & {$[.0000, .0002]$} \\
$\mathrm{W}$ & -.4049 & .0849 & .0000 & {$[-.5713,-.2385]$} \\
Sex & .6328 & .1218 & .0000 & {$[.3940, .8715]$} \\
ccons & -11.5778 & .6708 & .0000 & {$[-12.8925,-10.2631]$} \\
\hline Random part & & & & \\
\hline$\sigma_{\mathrm{A}}$ & 1.3355 & .2688 & & {$[.9002,1.9813]$} \\
$\sigma_{\mathrm{E}}$ & 1.1302 & .2871 & & {$[.6870,1.8594]$}
\end{tabular}

$\mathrm{AIC}=8863.3520(\mathrm{df}=11)$

\section{Table A3}

AE Model With Other Traumatic SLEs and Only Threshold MDE Diagnoses

\begin{tabular}{lcccl} 
Fixed part & $\beta$ & $S E$ & $\mathrm{p}$ & $95 \%$ Confidence Interval \\
\hline othtrauma_cm & .6536 & .1964 & .0010 & {$[.2686,1.0385]$} \\
othtrauma_cm\#c.dz & -.0846 & .2395 & .7240 & {$[-.5540, .3849]$} \\
othtrauma_cmc & -.1067 & .2791 & .7020 & {$[-.6538, .4404]$} \\
othtrauma_cmc\#c.dz & .0846 & .2395 & .7240 & {$[-.3849, .5540]$} \\
$\mathrm{t}$ & .4640 & .0865 & .0000 & {$[.2944, .6336]$} \\
$\mathrm{t}^{2}$ & -.0118 & .0036 & .0010 & {$[-.0189,-.0046]$} \\
$\mathrm{t}^{3}$ & .0001 & .0000 & .0270 & {$[.0000, .0002]$} \\
$\mathrm{W}$ & -.3971 & .0850 & .0000 & {$[-.5637,-.2305]$} \\
Sex & .7556 & .1269 & .0000 & {$[.5069,1.0043]$} \\
cons & -11.8776 & .6785 & .0000 & {$[-13.2074,-10.5478]$} \\
\hline Random part & & & & \\
\hline$\sigma_{\mathrm{A}}$ & 1.5121 & .2893 & & {$[1.0393,2.2000]$} \\
$\sigma_{\mathrm{E}}$ & 1.2151 & .3012 & & {$[.7476,1.9751]$}
\end{tabular}

$\mathrm{AIC}=8899.9360(\mathrm{df}=11)$ 
Table A4.

AE Model With Economic SLEs and Only Threshold MDE Diagnoses

\begin{tabular}{lcccl} 
Fixed part & $\beta$ & $S E$ & $\mathrm{p}$ & $95 \%$ Confidence Interval \\
\hline economic_cm & .5161 & .2382 & .0300 & {$[.0492, .9830]$} \\
economic_cm\#c.dz & .1653 & .3112 & .5950 & {$[-.4447, .7753]$} \\
economic_cmc & .8615 & .3175 & .0070 & {$[.2392,1.4837]$} \\
economic_cmc\#c.dz & -.1653 & .3112 & .5950 & {$[-.7753, .4447]$} \\
$\mathrm{t}$ & .4706 & .0866 & .0000 & {$[.3008, .6403]$} \\
$\mathrm{t}^{2}$ & -.012 & .004 & .0010 & {$[-.0192,-.0049]$} \\
$\mathrm{t}^{3}$ & .0001 & .0000 & .0230 & {$[.0000, .0002]$} \\
$\mathrm{W}$ & -.4010 & .0851 & .0000 & {$[-.5678,-.2343]$} \\
Sex & .6897 & .1250 & .0000 & {$[. .4448, .9346]$} \\
cons & -11.8536 & .6781 & .0000 & {$[-13.1827,-10.5245]$} \\
\hline Random part & & & & \\
\hline$\sigma_{\mathrm{A}}$ & 1.5263 & .2886 & & {$[1.0536,2.2111]$} \\
$\sigma_{\mathrm{E}}$ & 1.1912 & .2985 & & {$[.7290,1.9465]$}
\end{tabular}

$\mathrm{AIC}=8895.7930(\mathrm{df}=11)$ 